\title{
Interference Mitigation for Coded MB-OFDM UWB
}

\author{
Chris Snow, Lutz Lampe, and Robert Schober \\ Department of Electrical \& Computer Engineering \\ University of British Columbia, Vancouver, Canada
}

\begin{abstract}
We consider the ECMA-368 Multiband Orthogonal Frequency Division Multiplexing (MB-OFDM) standard for high rate Ultra Wideband (UWB) wireless communication in the 3.1$10.6 \mathrm{GHz}$ band. The performance of MB-OFDM is impacted by interference from IEEE 802.16 WiMAX systems operating in the licensed $3.5 \mathrm{GHz}$ band. Motivated by recent work showing the approximately Gaussian nature of the WiMAX interference to MB-OFDM, we propose a simple two-stage interference mitigation technique for coded MB-OFDM transmissions according to the ECMA-368 standard, consisting of interference spectrum estimation during silent periods followed by appropriate bit metric weighting during Viterbi decoding. We compare parametric and non-parametric spectrum estimation techniques for coded MB-OFDM transmissions and WiMAX interference for various scenarios of interest. The proposed two-stage interference mitigation technique is shown to be highly effective at mitigating the impact of WiMAX interference.
\end{abstract}

\section{INTRODUCTION}

In this paper, we consider the ECMA-368 Multiband Orthogonal Frequency Division Multiplexing (MB-OFDM) standard for high rate Ultra Wideband (UWB) wireless communication in the $3.1-10.6 \mathrm{GHz}$ band [1], [2]. Because UWB systems in this band are operating as spectral underlay systems [3], [4], they will unavoidably be impacted by the transmissions of incumbent systems. We consider as interferer the OFDM-based WiMAX IEEE 802.16 system for wireless metropolitan area networks, operating in the licensed $3.5 \mathrm{GHz}$ band [5].

When WiMAX is deployed in the $3.5 \mathrm{GHz}$ band, it will be a source of interference for MB-OFDM systems. For this reason, there has recently been great interest in coexistence techniques between WiMAX and UWB systems [6], [7]. In recent work [8], we investigated the effect of a WiMAX system operating in the $3.5 \mathrm{GHz}$ band and causing interference to a MB-OFDM system. In particular, we provided an exact analysis of the effect of the WiMAX system on the uncoded bit error rate (BER) of the MB-OFDM system, and compared the exact analysis with a Gaussian approximation for the WiMAX interference signal.

Motivated by the approximately Gaussian nature of the WiMAX interference [8], in this paper we propose a simple

The completion of this research was made possible thanks to Bell Canada's support through its Bell University Laboratories R\&D program and the Natural Sciences and Engineering Research Council of Canada (Grant CRDPJ 320 552), and with the support of a Canada Graduate Scholarship. This research was enabled by WestGrid computing resources, funded in part by the Canada Foundation for Innovation, Alberta Innovation and Science, BC Advanced Education, and participating institutions. Equipment is provided by IBM, HP, and SGI. Email: \{ csnow, Lampe, rschober \}@ece.ubc.ca two-stage interference mitigation technique for coded MBOFDM transmissions according to the ECMA-368 standard, consisting of interference spectrum estimation during silent periods followed by appropriate bit metric weighting during Viterbi decoding. We compare parametric and non-parametric spectrum estimation techniques for coded MB-OFDM transmissions and WiMAX interference for various scenarios of interest. The proposed two-stage interference mitigation technique is shown to be highly effective at mitigating the impact of WiMAX interference.

Organization: The remainder of this paper is organized as follows. In Section II, we introduce the necessary details of the MB-OFDM and WiMAX systems. The interference estimation and mitigation techniques are described in Section III. Numerical results illustrating the performance of the proposed techniques are given in Section IV, and Section V concludes the paper.

\section{PRELIMINARIES}

In this section we provide the necessary information about the MB-OFDM and WiMAX systems. Interested readers are referred to the relevant standards for further details [1], [5].

\section{A. MB-OFDM System}

The ECMA-368 MB-OFDM system [1] consists of OFDM with $N_{m}=128$ subcarriers and a bandwidth of $528 \mathrm{MHz}$, combined with frequency hopping over a number of subbands within the 3.1-10.6 GHz band (one hop per OFDM symbol). In this paper, we will assume the first-generation hopping pattern of three subbands, with center frequencies of 3432, 3960, and $4488 \mathrm{MHz}$, is employed. Note that, because of frequency hopping, only every third MB-OFDM symbol is impacted by the WiMAX interference in the $3.5 \mathrm{GHz}$ band. After the inverse fast Fourier transform, a guard interval of $70.07 \mathrm{~ns}$ (37 samples) is applied. The OFDM symbol duration is $312.5 \mathrm{~ns}$, of which $D_{m}=242.43 \mathrm{~ns}$ is for data.

Error correction coding consists of a punctured maximumfree distance rate 1/3 constraint length 7 convolutional code. Coded bits are interleaved before being modulated using Quaternary Phase Shift Keying (QPSK) modulation on each OFDM subcarrier. Thus, the MB-OFDM system consists of rather classical bit-interleaved coded modulation (BICM) [9] combined with frequency-hopping OFDM.

For a meaningful performance analysis of the Multiband OFDM proposal, we consider the channel model developed under IEEE 802.15 for UWB systems [10]. The channel impulse response is a Saleh-Valenzuela model modified to fit 
the properties of measured UWB channels. Multipath rays arrive in clusters with exponentially distributed cluster and ray interarrival times. Both clusters and rays have decay factors chosen to meet a given power decay profile. The ray amplitudes are modeled as lognormal random variables, and each cluster of rays also undergoes a lognormal fading. To provide a fair system comparison, the total multipath energy is normalized to unity. Finally, the entire impulse response undergoes an "outer" lognormal shadowing. The channel impulse response is assumed time invariant during the transmission period of several packets (see [10] for a detailed description).

\section{B. WiMAX System}

The OFDM-based WiMAX IEEE 802.16 system [5] employs $N_{n}=256$ subcarriers, and supports Binary Phase Shift Keying (BPSK), QPSK, 16QAM (Quadrature Amplitude Modulation), and 64QAM modulation schemes. We will focus on QPSK modulation in this paper, but similar results are observed for the other modulation schemes. The system supports variable carrier frequency $f_{n}$ (we focus on the $3.5 \mathrm{GHz}$ band), as well as various operating bandwidths.

\section{INTERFERENCE ESTIMATION AND MITIGATION FOR CODED MB-OFDM}

It is natural to seek means to mitigate the impact of WiMAX interference on MB-OFDM systems. Recent results have shown that the MB-OFDM per-subcarrier interferenceplus-noise distribution in the presence of WiMAX interference behaves in an approximately Gaussian manner [8]. Given the near-Gaussian nature of these per-subcarrier interference statistics, one natural and near-optimum technique for interference mitigation is to (1) estimate the per-subcarrier interference-plus-noise power, and (2) use this information to weigh the branch metrics fed to the Viterbi decoder, in order to suppress the interference effects. This particular technique requires only modest increases in receiver complexity, and does not require any modifications to the MB-OFDM transmitter or signal structure. We describe each stage of the process below.

\section{A. Interference Estimation}

The MB-OFDM system will listen to the channel for interference estimation purposes, either (a) during the silent period between packet transmissions, or by (b) listening to one subband while operating on another sub-band. Furthermore, we assume that no other UWB devices transmit in the considered sub-band during the silent time, so that the receiver will detect only the interference-plus-noise that exists in the channel. We let $P$ be the number of MB-OFDM symbol durations that are used to observe each sub-band.

We consider two methods for spectral estimation, described below. Both approaches adopt a time-domain estimation followed by a Fourier transform to obtain the final per-subcarrier noise variance estimates. Time-domain estimation allows us to exploit the limited degrees of freedom in the interference signal. We denote the resultant interference-plus-noise variance estimate for subcarrier $k$ by $\hat{S}\left(\omega_{k}\right)$, where $\omega_{k}=2 \pi k / N_{\mathrm{m}}$.
1) Parametric Approach: We first adopt a parametric approach by fitting the time-domain silent period observations to an autoregressive (AR) model. The fitting method is that of maximum entropy, also known as the Burg method [11]. For a given AR model order $M$, the Burg spectral estimate is given by

$$
\hat{S}_{\text {Burg }}(\omega)=\frac{P_{M}}{\left|1+\sum_{i=1}^{M} a_{M, i} e^{-j i \omega}\right|^{2}},
$$

where $P_{M}$ and $a_{M, i}$ are the parameters of the AR model, obtained with the Levinson-Durbin algorithm [11, Sec. 9.5, pp. 414-420]. The parametric approach is generally able to use small estimation periods $P$. However, the performance of the method is dependent on a proper choice of model order $M-$ smaller model orders allow for simpler estimators, but may not yield suitable estimates in the presence of multiple interferers or other complicated interference scenarios.

2) Non-parametric Approach: We also consider a nonparametric approach to spectral estimation. We adopt the multi-taper method (MTM) [11], advocated for use in radioscene analysis for cognitive radio [12]. In the MTM, a set of orthogonal windows (or tapers) $w_{i}(n)$ are applied to the observed data and the resultant estimates are averaged. In this work we adopt tapers based on the Slepian (or discrete prolate spheroidal) sequences [11, Chap. 8], which have maximal energy concentration for finite bandwidth and sample size. We use $P$ tapers for a window of $P$ MB-OFDM observation symbols, for a total of $N=(128+37) P$ samples taken at rate $T=1 /\left(528 \times 10^{6}\right) \mathrm{s}$. The MTM spectral estimate of the discrete-time observed signal $b(n T)$ is given by

$$
\hat{S}_{\mathrm{MTM}}(\omega)=\frac{1}{P} \sum_{i=0}^{P-1}\left|\sum_{n=0}^{N-1} w_{i}(n) b(n T) e^{-j \omega n T}\right|^{2} .
$$

\section{B. Interference Mitigation}

The estimators given above predict the interference seen at the input to the MB-OFDM receiver FFT. However, we will mitigate the interference during decoding, after the FFT, and thus must account for the effect of the rectangular time-domain window of length $D_{\mathrm{m}}$. The spectrum after windowing is given by

$$
\hat{S}_{W}(\omega)=\hat{S}_{X}(\omega) \otimes\left[\frac{\sin \left(\omega D_{\mathrm{m}}\right)}{\omega}\right]^{2},
$$

with $X \in\{$ Burg, MTM $\}$, and where $\otimes$ denotes the convolution operator.

As mentioned, the MB-OFDM system employs BICM for error protection. Decoding consists of soft de-mapping, followed by de-interleaving and Viterbi decoding. Given that the interference-plus-noise per subcarrier is approximately Gaussian, we maintain the standard Euclidean distance metric, and scale the branch metrics for all bits from subcarrier $k$ by $1 / \hat{S}_{W}\left(\omega_{k}\right)$. The effect of the correlation between adjacent subcarriers is negligible due to the de-interleaving process, and can be neglected. 


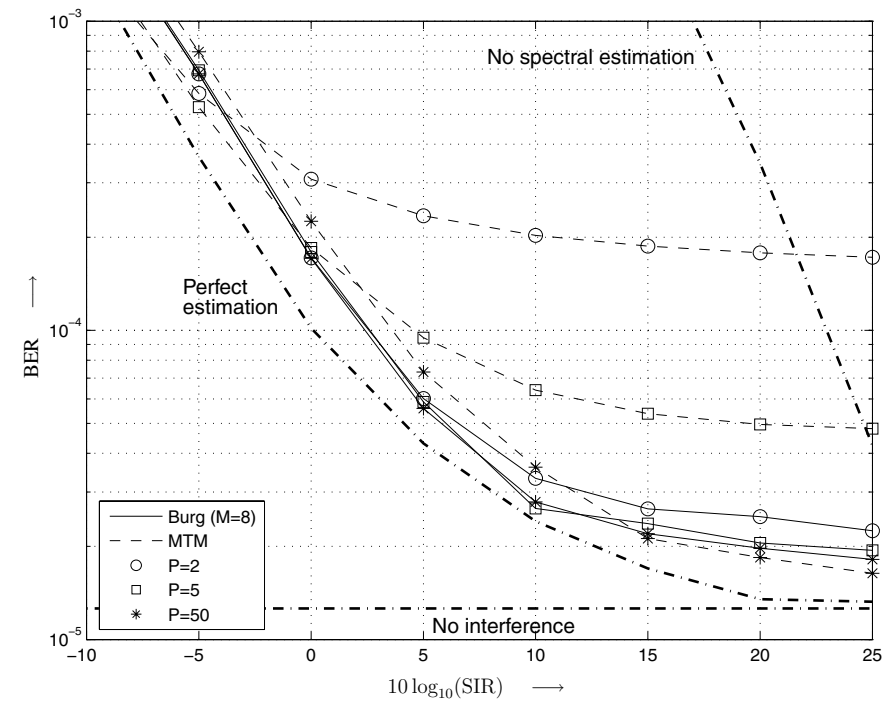

Fig. 1. BER versus $10 \log _{10}(\mathrm{SIR})$ for $10 \log _{10}(\mathrm{SNR})=14.5$. MBOFDM coded transmission with rate $1 / 2$. WiMAX bandwidth $7 \mathrm{MHz}$, carrier frequency $f_{n}=3500 \mathrm{MHz}$. UWB CM1 channel, non-fading WiMAX channel. Burg with $M=8$ (solid lines) and MTM (dashed lines) spectral estimation techniques, for $P \in\{2,5,50\}$ symbols. For comparison: BER with no spectral estimation, perfect spectral estimation, and no interference (thick dash-dotted lines).

Note that, in the presence of purely Gaussian noise, the strategy described above is optimal. We do not simply discard information from subcarriers, but rather reduce the influence of bits which have been impacted by WiMAX interference. We note that erasure decoding (as proposed in e.g. [13]) can be seen as a special case of this technique when $\hat{S}_{W}\left(\omega_{k}\right) \rightarrow \infty$ for some $k$.

\section{NUMERICAL RESUlts}

In this section we present results for coded MB-OFDM systems employing the interference estimation and mitigation technique discussed in Section III. Because we want to isolate the effects of the interference mitigation, we focus on nonfading WiMAX channels, and note that similar behaviors will be observed with fading channels. We also assume the WiMAX system is continually transmitting, i.e., we do not consider arrival/departure of WiMAX systems during the transmission interval. Interference estimation is performed anew before each MB-OFDM data packet transmission, and the estimates are then fixed for the duration of the MB-OFDM packet.

In Figure 1, we plot the MB-OFDM BER versus the $10 \log _{10}$ (SIR) (signal to interference ratio) for coded transmission with rate $1 / 2$ and $10 \log _{10}(\mathrm{SNR})=14.5$ (signal to noise ratio). For the MB-OFDM system, we adopt the UWB channel model CM1 [10], and average over 500 channel realizations. The interferer is a WiMAX system with bandwidth $7 \mathrm{MHz}$ and carrier frequency $f_{n}=3500 \mathrm{MHz}$. We consider both the Burg with $M=8$ (solid lines) and MTM (dashed lines) spectral estimation techniques, for $P \in\{2,5,50\}$ symbols.

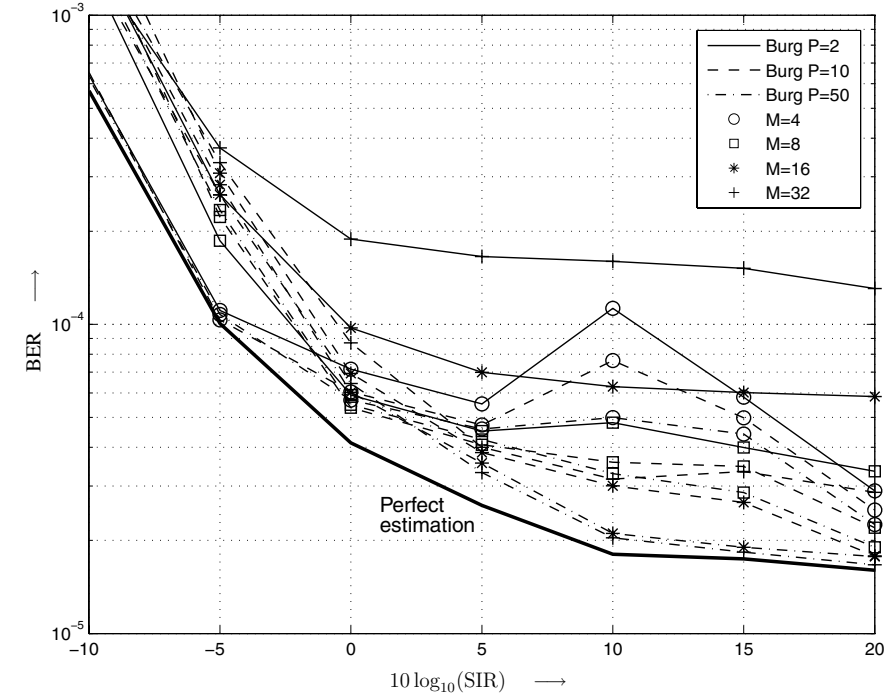

Fig. 2. BER versus $10 \log _{10}(\mathrm{SIR})$ for $10 \log _{10}(\mathrm{SNR})=4.1$ MB-OFDM coded transmission with rate $1 / 2$. WiMAX bandwidth $1.75 \mathrm{MHz}$, carrier frequency $f_{n}=3500 \mathrm{MHz}$. Non-fading WiMAX and MB-OFDM channels. Burg spectral estimation technique, AR model orders $M \in\{4,8,16,32\}$ and $P \in\{2,5,50\}$ symbols. For comparison: perfect spectral estimation (thick solid line).

For comparison we also include the MB-OFDM BER with no spectral estimation, perfect spectral estimation, and no interference (thick dash-dotted lines). The perfect spectral estimation curves are obtained by assuming the receiver has perfect knowledge of the noise variance $\sigma_{n}^{2}$ and interference variances $\sigma_{i, k}^{2}$ (given in [8]) when calculating the branch metric weights as described in Section III-B.

We make several observations about the results in Figure 1. Firstly, for small numbers of estimation symbols $P \in\{2,5\}$ the MTM estimation technique performs poorly, because such small observation lengths are not sufficient to provide reliable non-parametric estimation. On the other hand, for $P=50$ observation symbols the MTM method is comparable to the parametric approach. Secondly, we observe that for low values of SIR, the Burg estimator performance is relatively invariant to the choice of $P$, while at higher SIR there are small gains with increasing $P$. Finally, we note that both the parametric (with $P=50$ ) and non-parametric approaches perform relatively close to the perfect estimation limit, and also provide substantial performance improvements in comparison with the case of no interference mitigation.

In Figure 2, we examine the effect of varying the Burg AR model order $M$. We adopt a non-fading MB-OFDM channel, code rate $1 / 2,10 \log _{10}(\mathrm{SNR})=4.1$, one WiMAX interferer with bandwidth $1.75 \mathrm{MHz}$, AR model orders $M \in$ $\{4,8,16,32\}$ and $P \in\{2,10,50\}$ estimation symbols. We can see that at low SIR all model orders have relatively similar performance, with $M=4$ providing slightly better performance than larger $M$. At higher SIR, increased model orders lead to better performance for $P=50$, but poor performance for the short estimation interval $P=2$. At intermediate values 


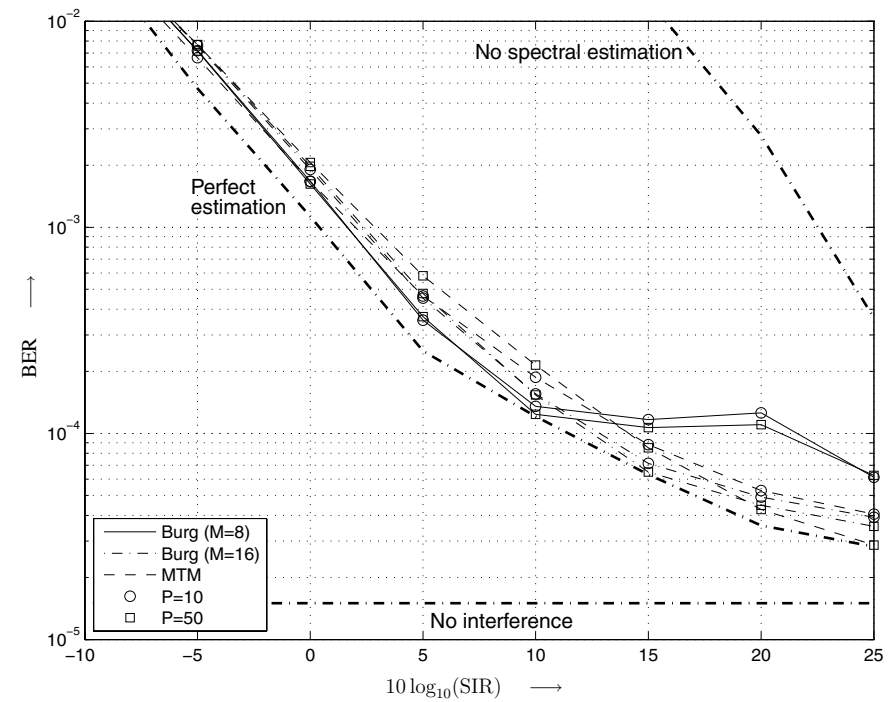

Fig. 3. BER versus $10 \log _{10}(\mathrm{SIR})$ for $10 \log _{10}(\mathrm{SNR})=18$. MBOFDM coded transmission with rate $3 / 4$. Two WiMAX interferers with bandwidth $7 \mathrm{MHz}$, carrier frequencies $f_{n}=\{3475,3500\}$ MHz. UWB CM1 channel, non-fading WiMAX channel. Burg with $M \in\{8,16\}$ (solid, dash-dotted lines) and MTM (dashed lines) spectral estimation techniques, for $P \in\{10,50\}$ symbols. For comparison: BER with no spectral estimation, perfect spectral estimation, and no interference (thick dash-dotted lines).

of SIR, model order $M=4$ is insufficient, with inaccurate modeling of the interference spectrum leading to degrading performance with increasing SIR. These results indicate the importance of choosing appropriate estimation parameters $M$ and $P$ in order to guarantee reasonable interference mitigation performance.

We consider a more complicated interference scenario in Figure 3, with two WiMAX systems operating at $3475 \mathrm{MHz}$ and $3500 \mathrm{MHz}$, each with a bandwidth of $7 \mathrm{MHz}$. The MBOFDM system operates over UWB channel CM1. We also consider a higher code rate of $3 / 4$, which provides less error protection to the transmitted MB-OFDM data. We can see that in this case, the Burg spectral estimator with $M=8$ does not perform well in the intermediate SIR range, due to the inability of the $M=8$ tap AR model to accurately represent the interference spectrum. On the other hand, both the MTM and Burg with $M=16$ perform well for all values of SIR. This result indicates that some consideration of the potential interference environment must be made in the design of interference mitigation techniques for MB-OFDM systems. In general, if larger values of $P$ can be tolerated, the MTM estimator may be preferable, while for smaller $P$ a Burg estimator with properly selected $M$ offers reasonable performance.

\section{Conclusions}

Coexistence and the ability to appropriately handle interference from incumbent narrowband systems are important aspects of the design of UWB devices. The particular example of WiMAX in the $3.5 \mathrm{GHz}$ band is of practical interest due to the potential for large-scale WiMAX deployment in the near future.

Motivated by the approximately Gaussian nature of WiMAX interference, we have presented a two-stage interference mitigation technique, consisting of interference spectral estimation followed by interference mitigation during Viterbi decoding. We have compared parametric and non-parametric approaches for several interference scenarios of practical interest. In the presence of WiMAX interference, the two-stage interference mitigation provides substantial gains in performance in return for modest increases in receiver complexity and without requiring any modifications to the MB-OFDM transmitter or signal structure. However, our results show that the expected interference environment should be carefully considered during the design of such mitigation techniques.

\section{REFERENCES}

[1] ECMA, "Standard ECMA-368: High Rate Ultra Wideband PHY and MAC Standard," Dec. 2005, [Online]: http://www.ecmainternational.org/publications/standards/Ecma-368.htm.

[2] A. Batra, J. Balakrishnan, G. Aiello, J. Foerster, and A. Dabak, "Design of a Multiband OFDM System for Realistic UWB Channel Environments," IEEE Trans. Microwave Theory Tech., vol. 52, no. 9, pp. 21232138 , Sept. 2004.

[3] Federal Communications Commission (FCC), "Revision of Part 15 of the Commissions Rules Regarding Ultra-Wideband Transmission Systems," First Report and Order, ET Docket 98-153, FCC 02-48; Adopted: February 14, 2002; Released: April 22, 2002.

[4] Q. Zhao and B. M. Sadler, "A Survey of Dynamic Spectrum Access," IEEE Signal Processing Mag., vol. 24, no. 3, pp. 79-89, May 2007.

[5] IEEE Std 802.16-2004, "Part 16: Air Interface for Fixed Broadband Wireless Access Systems," Oct. 2004.

[6] V. Somayazulu, J. Foerster, and R. Roberts, "Detect and Avoid (DAA) Mechanisms for UWB Interference Mitigation," in Proc. IEEE Intl. Conf. on Ultra-Wideband (ICUWB), Waltham, MA, USA, Sept. 2006, pp. 513518.

[7] A. Durantini, R. Giuliano, F. Mazzenga, and F. Vatalaro, "Performance Evaluation of Detect and Avoid Procedures for Improving UWB Coexistence with UMTS and WiMAX systems," in Proc. IEEE Intl. Conf. on Ultra-Wideband (ICUWB), Waltham, MA, USA, Sept. 2006, pp. 501506.

[8] C. Snow, L. Lampe, and R. Schober, "Analysis of the Impact of WiMAX-OFDM Interference on Multiband OFDM," in Proc. IEEE Intl. Conf. on Ultra-Wideband (ICUWB), Singapore, Sept. 2007.

[9] G. Caire, G. Taricco, and E. Biglieri, "Bit-Interleaved Coded Modulation," IEEE Trans. Inform. Theory, vol. 44, no. 3, pp. 927-946, May 1998.

[10] A. F. Molisch, J. R. Foerster, and M. Pendergrass, "Channel Models for Ultrawideband Personal Area Networks," IEEE Wireless Commun. Mag., pp. 14-21, Dec. 2003.

[11] D. B. Percival and A. T. Walden, Spectral Analysis for Physical Applications: Multitaper and Conventional Univariate Techniques. Cambridge University Press, 1993.

[12] S. Haykin, "Cognitive Radio: Brain-Empowered Wireless Communications," IEEE J. Select. Areas Commun., vol. 23, no. 2, pp. 201-220, Feb. 2005.

[13] T. Li, W. H. Mow, V. K. Lau, M. Siu, R. S. Cheng, and R. D. Murch, "Robust Joint Interference Detection and Decoding for OFDM-Based Cognitive Radio Systems with Unknown Interference," IEEE J. Select. Areas Commun., vol. 25, no. 3, pp. 566-575, Apr. 2007. 